\title{
Impact of Dextran Biodegradation Catalyzed by Dextranase Enzyme on the Crystallization Rate of Sucrose during Sugar Manufacturing
}

\author{
Mohanad Bashari ${ }^{1, *}$, Camel LAGNIKA ${ }^{2}$, Al-farga Ammar ${ }^{3}$, Mandour H. Abdalhai ${ }^{4}$, Ayman Balla Mustafa ${ }^{5}$ \\ ${ }^{1}$ A'Sharqiah University, College of Applied and Health Sciences, Dept. of Food Science and Human Nutrition, \\ P.O.Box 42 Postal Code 400, Ibra, Sultanate of Oman. \\ ${ }^{2}$ School of Sciences and Techniques for Preservation and Processing of Agricultural Products, \\ National University of Agriculture, BP: 114 Sakete, Republic of Benin. \\ ${ }^{3}$ College of Sciences, Biochemistry Department, University of Jeddah, Saudi Arabia \\ ${ }^{4}$ Jiangsu University, School of Food and Biological Engineering, China \\ ${ }^{5}$ Therapeutic Nutrition Department, Faculty of Nursing and Health Sciences, Misrata University, Libya \\ *Corresponding author: mohanad.bashari@asu.edu.om
}

Received April 03, 2019; Revised May 11, 2019; Accepted May 21, 2019

\begin{abstract}
Introduction: In this research work, we investigated the influence of the biodegradation of dextran catalyzed by dextranase enzymes during sugar manufacturing on the rate of sucrose crystallization and growth rate of sucrose crystals in pure sucrose solution at different temperatures. Methods: To elucidate the influence of biodegradation of dextran on the growth rate of sucrose crystals, dextran of $\mathrm{Mw} 2,000,000 \mathrm{~g} / \mathrm{mol}\left(\mathrm{T}_{2000}\right)$ was admixed in concentrations between (1000 - $10000 \mathrm{ppm})$ with $(60 \%-75 \% \mathrm{w} / \mathrm{w})$ sucrose solution.. The hydrolysis of dextran was carried out at $55.0^{\circ} \mathrm{C}$ and $\mathrm{pH} 5.5$ at different dextranase concentration, and then the samples were immediately subjected to the crystallization process. Results: The most pronounced effect of dextran on the growth rate of sucrose crystals was found with T2000 at concentrations more than $5000 \mathrm{ppm}$ at $60^{\circ} \mathrm{C}$. From the results it could be shown that an increase of crystallization rate of up to $50 \%$ after biodegradation of dextran $\mathrm{T}_{2000}$ using dextranase enzyme at concentration of $100 \mathrm{ppm}$, compared to crystallization rate with pure sucrose solution in the presence of dextran $\mathrm{T}_{2000}$. It was obvious that after dextran hydrolyzed by dextranase, more perfect crystal surfaces are built than at $60^{\circ} \mathrm{C}$. Conclusion: Dextran biodegradation catalyzed by dextranase enzyme has increased the crystallization rate of sucrose and more perfect crystal surfaces are built. Such a positive influence of biodegradation of dextran using dextranase enzyme decreases crystallization time in the sugar house and thus decreases the production costs of sugar manufacturing.
\end{abstract}

Keywords: dextranase, dextran, sugar manufacturing, sucrose crystallization, growth rate, enzymatic treatment

Cite This Article: Mohanad Bashari, Camel LAGNIKA, Al-farga Ammar, Mandour H. Abdalhai, and Ayman Balla Mustafa, "Impact of Dextran Biodegradation Catalyzed by Dextranase Enzyme on the Crystallization Rate of Sucrose during Sugar Manufacturing.” Journal of Food and Nutrition Research, vol. 7, no. 5 (2019): 402-408. doi: 10.12691/jfnr-7-5-10.

\section{Introduction}

In a sugar factory, the crystallization process is an important step, which determines the yield of the sugar from sugar beet and cane. Meade and Chen reported that the rate of crystallization was affected by the degree of supersaturation, temperature, crystal surface area, and nature or concentration of impurities [1]. In fact, the crystal growth rate is also affected by high molecular mass substances and colorants in syrups, which are partially adsorbed on the crystal surface and thus impede the insertion of sucrose molecules into the lattice [2].

Dextrans are high molecular weight polysaccharides, formed of at least $50 \%$, by $\alpha-(1-6)$ linked glucose units, with $\alpha-(1-3)$ branch linkages and may contain other branch linkages such as $\alpha-(1-2)$ or $\alpha-(1-4)$ [3]. Dextran is produced by micro-organisms such as Leuconostoc mesenteroides that contaminate sugar cane in the rainy seasons [1] and it is produced by bacterial fermentation in medium that is rich in sucrose. These dextrans are extracted in the mills along with the juices and contaminate the sugar mill flow, reaching levels in the juice exceeding 10,000 ppm (1\%) in very extreme cases. In burnt sugar cane a rapid increase in the level of dextrans of almost ten times was observed from 12 to $48 \mathrm{~h}$, reaching $3200 \mathrm{ppm}$ [4]. The number of branches and the molecular weight of dextran are its key properties and define the behaviour of these compounds in solutions [5].

The presence of dextran in the sugar factories leads to a falsely high polarization, increased viscosity, slowing of 
filtration, lower evaporation rates, elongated crystals (needle grain), longer wash and separation cycles in centrifuges and increase of sugar loss to molasses [6]. The most damaging effects of elevated dextran concentrations in a technical sucrose solution are foreseen in the crystallization process. Dextrans slow down the crystallization rate or even inhibit crystallization [7]. Recently, Eggleston et al., suggested that high Mw dextran may have contributed to the hard-to-boil phenomenon which occurs in some massecuite samples [8].

Dextranase (1,6- $\alpha$-D-glucan-6-glucanohydrolase, EC3.2.1.11), is an enzyme, which catalyzes endohydrolysis of $\alpha$-(1-6)-d-glycoside linkages in random sites of dextran [3]. The application of dextranases in the sugar industry was pioneered in Australia in the 1970s. In South Africa where diffusers are used, dextranase application in diffuser cane juices was deemed unsuitable mostly because of the high temperatures [9]. Recently, new methods have been reported to enhance the enzymatic hydrolysis of dextran catalyzed by dextranase using intensity ultrasonic combined with high hydrostatic pressure or microwave Irradiation which could be an effective methods for improving the industrial efficiency of dextranases in many industrial applications including sugar manufacturing processes [10,11,12].

The main focus of this research work was to investigate the effects of hydrolysis of dextran using dextranase enzyme on the crystallization process and the quality of the final sugar crystal. Moreover, the effects of addition of dextranase to sugar juices as industrially relevant method on growth rate of sucrose crystals, crystal shape and surface topography at different crystallization temperature and supersaturation were also identified.

\section{Materials and Methods}

\subsection{Materials}

Dextranase produced by Chaetomium erraticum, dextran $\mathrm{T}_{2000} \mathrm{Mw} \sim 2,000,000$ ) used to increase the polysaccharides content of the sucrose solutions, and Sucrose crystals $d=200 \mu \mathrm{m}$ used as seed, were obtained from Sigma-Aldrich (Shanghai, China). Analytical grade refinery fine sucrose was obtained from Sinopharm Chemical reagent Co. (Shanghai, China). All other chemicals and solvents used were of analytical grade.

\subsection{Preparation of Sucrose Solution and Dextran}

Sucrose solutions with concentrations of $65 \%$ and $75 \%$ (w/w) were prepared by weighing the analytical grade sucrose and dissolved in the corresponding amount of distilled water. The mixture was then heated in a microwave oven (medium power) for short periods (45 sec), intercalated with agitation until to complete the sucrose dissolution [13]. The use of microwave heating, which results in high temperatures for short time periods, is based on the fact that sucrose in concentrated solutions presents a lag phase in the thermal degradation reaction [14] and will not undergo any degradation during solutions preparation. To confirm that no sucrose degradation occurred during the preparation of the solutions, a sample of each solution was analyzed using HPLC system [15]. It was found that no significant sucrose degradation has occurred during the preparation of the samples.

The present studies have been done by the step-wise addition of dextran at a rate of 1000 ppm (0.1\%) up to a maximum of 10000 ppm (1\%), as this quantity of dextran was found to exist in cane sugar process streams. In order to avoid damage or thermal degradation due to the heating process on dextran at different $\mathrm{Mw}$ and concentrations, initially dextran was dissolved in cold water and then the polysaccharide solutions were added to the sucrose solutions and mixed using a magnetic stirrer to give the desired concentrations.

\subsection{Determination of Dextran}

Dextran concentration for all samples was determined according to ICUMSA GS1-15 (1994) and rapid haze [16] methods with some modifications to improve the accuracy. Sucrose solution $(3 \mathrm{ml})$ was added to $3 \mathrm{ml}$ absolute ethanol, mixed and left for $2 \mathrm{~min}$. The absorbance at $720 \mathrm{~nm}$ was immediately read in a 1-cm cell on a UV-1800 spectrophotometer (Shanghai Mapada Instruments Co., Ltd, China). Dextran $\left(\mathrm{T}_{2000}\right)$ was used to prepare the standard and dextran was precipitated with $100 \%$ absolute ethanol.

\subsection{Enzymatic Hydrolysis of Dextran in the Sucrose Solution}

Dextranase enzyme (at concentrations between 0 -100 ppm) was added to $100 \mathrm{~mL}$ sucrose solution containing dextran (10.000 ppm) in flask, covered with aluminum foil, and thoroughly mixed. For the control, de-ionized water was added instead of enzyme. The glasses flasks were placed in a shaking water-bath (120 rpm) at $50^{\circ} \mathrm{C}$ for $30 \mathrm{~min}$. After incubation, the samples were immediately subjected to the crystallization process.

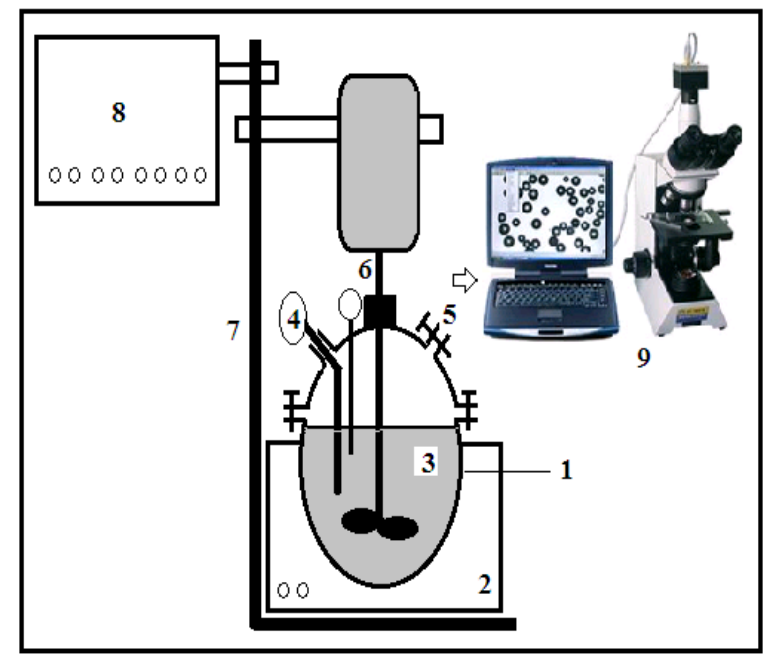

Figure 1. Pilot scheme of laboratory crystallizer equipped: 1 . double wall glass crystallizer 2 . water bath 3 . automatic stirrer 4 . refractometer 5. samples collection part 6 . thermometer 7. carrier 8. control panel 9. electronic microscope 


\subsection{Crystallization Unit}

The scheme of laboratory crystallization unit is given in Figure 1. The experimental apparatus used in this study consisted of a glass crystallizer equipped with automatic stirrer, Refractometer, and thermometer. The temperature in the crystallizer was controlled by means of a heating bath equipped with an external temperature sensor which was set in the solution. Dry substance and temperature were determined every $120 \mathrm{~s}$.

\subsection{Crystallization Experiments}

For our research work the isothermal crystallization method was used to study the effect of hydrolysis of dextran using dextranase enzyme on the crystallization process without interference of other factors. Crystallization process was performed at constant temperatures (60, and $70^{\circ} \mathrm{C}$ ). The agitation inside the crystallizer was maintained at a constant speed of $200 \mathrm{rpm}$. After reaching the required temperature, the solution in the crystallizer was seeded with sucrose crystals (size $200 \mu \mathrm{m}$ ) using a syringe. The amount of seed $m_{\text {Seed }}$ in $g$ was calculated as follows [7]:

$$
m_{\text {seed }}=m_{M a} * w_{C r y} *\left(d_{i} / d_{f}\right)^{3}
$$

Where, $\mathrm{m}_{\mathrm{Ma}}$ is the massecuite mass in $\mathrm{g}, \mathrm{w}_{\text {Cry }}$ is the final crystal content in massecuite, $d_{i}$ is the initial size of the crystal $(200 \mu \mathrm{m})$ and $\mathrm{df}$ is the final size of the crystal.

A sample was taken before the seeding point to carry out the first image analysis for the evaluation of the solubilization process. The kinetic measurements were obtained by periodically removing samples of the solution from the crystallizer and placing them under the microscope for image capture in order to follow the growth of crystals through the crystallization. Solution concentration was measured with the refractometer. To remove any particles sticking to the crystal surface, the crystals were washed in a slightly subsaturated solution, then dabbed with filter paper and dried in an airstream.

\subsection{Calculation of the Growth Rate and Surfaces Area of Sucrose Crystals}

The growth rate of sucrose crystals $(G)$ in $g /(m 2$ min) was calculated later as follows:

$$
G=\left(\frac{\Delta m_{s, C r y}}{t}\right) *\left(\frac{1}{A_{C r y}}\right)
$$

Where $m_{s, \text { Cry }}$ is the crystallized sucrose mass, $t$ is the time (min), and $A_{C r y}$ is the total crystal surfaces in $\mathrm{m}^{2}$. The crystallized sucrose mass $\left(\mathrm{m}_{\mathrm{S} \text {, Cry }}\right)$ was calculated according to this equation:

$$
\begin{aligned}
\Delta m_{S, C r y}= & \left(\frac{w_{D S, M S, t}}{100-w_{D S, M S, t}} \cdot m w\right) \\
& -\left(\frac{w_{D S, M S, t+1}}{100-w_{D S, M S, t+1}} \cdot m w\right)
\end{aligned}
$$

Where $\mathrm{W}_{\mathrm{DS}}$ is dry substrate content in \%, mw is the mass of water, $\mathrm{M}_{\mathrm{S}}$ is the mother syrup.
Sucrose crystal surfaces area (ACry) was calculated using the following equation:

$$
A_{\text {Cey }}=f_{A} * m_{\text {Cry }}^{\frac{2}{3}} * n_{C e y}
$$

Where, $\mathrm{f}_{\mathrm{A}}$ form factor $(0.0423), \mathrm{m}_{\text {Cry }}$ is the crystal mass and $\mathrm{n}_{\text {Cry }}$ is the number of crystals [17].

\subsection{Scanning Electron Microscopy (SEM) Analysis}

A scanning electronic microscope (SEM) was used to examine the surface morphology of all the samples. A double-sided tape was first attached to specimen holder then the tape was slightly sprinkled with sample powder. Specimens were coated with a gold-palladium layer using SEM sputter coater. They were examined under the SEM (QUANTA 200F, FEI, Netherlands) at an accelerating voltage of $5.0 \mathrm{kV}$ and a working distance of 8.0 or $8.2 \mathrm{~mm}$.

\subsection{Statistical Analysis}

All the experiments described above, were performed in triplicate for each sample. The data was subjected to an analysis of variance (ANOVA) and the significance of the difference between means was determined by Duncan's multiple range test $(\mathrm{P}<0.05)$ using SPSS 17 for Windows program (SPSS Inc., Chicago, USA).

\section{Results and Discussion}

\subsection{Enzymatic Biodegradation of Dextran}

In our previous research work, we investigated the influence of dextranase enzyme on the molecular weight parameters of remaining dextran and intrinsic viscosity after different enzymatic treatments at different steps during sugar manufacturing [18]. Addition of dextranase to juice was much more efficient and economical to reduce the $\mathrm{Mw}$ of remaining dextran than adding it to evaporator syrups. Addition of dextranase at juice $\mathrm{pH} 5.5$ showed similar minimum $\mathrm{Mw}$ with the lowest intrinsic viscosity, observed at $55.0^{\circ} \mathrm{C}$. The highest dextran removal was observed at dextranase concentration of 100 $\mathrm{ppm} /$ juice which resulted in 80.29 \% removal dextran in the juice. Moreover, the higher the level of concentrated dextranase applied to the juice, the more the removal of dextran occurred [19]. In addition, the longer the availability of the residence time in the factory the lower dextran Mw has been observed. Therefore, in this study the hydrolysis of dextran was carried out at $55.0^{\circ} \mathrm{C}$ and $\mathrm{pH}$ 5.5 at different dextranase concentration, and then the samples were immediately subjected to the crystallization process.

\subsection{Influence of Dextran Biodegradation by Dextranase on Growth Rate of Sucrose Crystals at a Constant Temperature}

The growth rates of sucrose crystals during the crystallization process for pure sucrose solution after 
hydrolysis of dextran $\mathrm{T}_{2000}$ by different dextranase concentration at $60^{\circ} \mathrm{C}$ are shown in Figure 2. The results indicated that indicates that the crystallization rate regarding a supersaturation of 1.13 in the sugar solution without dextran (control) is the same as the crystallization rate at supersaturations of 1.135 and 1.138 in presence of 1500 and 2000 ppm of dextran $T_{2000}$, respectively. These results show the same tendency as reported before $[1,7,20]$. However, growth rates obtained in own experiments were higher than the former results of Abdel-Rahman. This is due to the use of a fast mixing system during our own experiments in contrast to the float bed (without stirrer) crystallizer that was used by Abdel-Rahman et al, [7]. Also, a stronger effect of dextranase concentration can be observed at $60^{\circ} \mathrm{C}$. As it can be seen from Figure 2, the crystallization rates at $60^{\circ} \mathrm{C}$ at a supersaturation of 1.13 are reduced by $34 \%$ in presence $5000 \mathrm{ppm}$ dextran $\mathrm{T}_{2000}$, However, after hydrolysis of dextran by dextranase at 50, 75, $100 \mathrm{ppm}$ concentration, the crystallization rates at $60^{\circ} \mathrm{C}$ at a supersaturation of 1.13 are reduced by 30.3 , $21.5,8.1 \%$, respectively. The above observation was due to the fact that the hydrolysis of dextran reduced the viscosity of the sucrose solution. In our previous work using laboratory trials performed by adding standard dextrans to pure sucrose solutions [18], we have demonstrated that, both the apparent viscosity and dynamic modulus increased with an increase in dextran concentrations and they demonstrated strong dependence on its Mw [19]. Conversely, it can be expected that the use of dextranase will reduce the contribution from dextran concentrations and $\mathrm{Mw}$, resulting in decreased viscosity and, presumably, better boiling house recoveries. However, several byproducts are associated with the formation of dextran. These include acetic acid, lactic acid, ethanol, mannitol and carbon dioxide as well as several oligosaccharides [8]. Many of these products are melassegenic and will also contribute to decreased exhaustion. Their influences will be unaffected by the dextranase treatment.

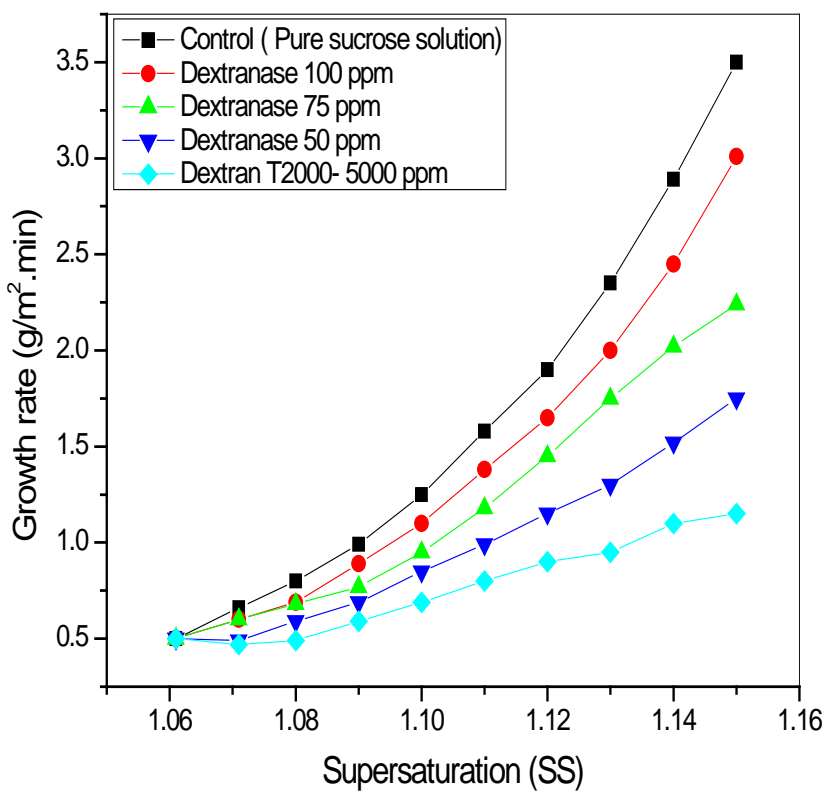

Figure 2. Growth rate of sucrose crystals at $60{ }^{\circ} \mathrm{C}$ after hydrolysis of dextran $\mathrm{T}_{2000}$ by different dextran concentration

\subsection{Influence of Hydrolysis of Dextran on by Dextranase on the Crystallization Time}

Figure 3 illustrates the relationship between the dry substance content and the time of the crystallization process for pure sucrose solutions (control) and after addition of dextran $\mathrm{T}_{2000}(5000 \mathrm{ppm})$ at $60 \mathrm{C}$. The presence of dextran increased the crystallization time compared with the pure sucrose solution (control), however, a decrease in the crystallization time after hydrolyses of dextran by different dextranase concentration was observed. An increase of dextranase concentration caused a decrease of crystallization time.

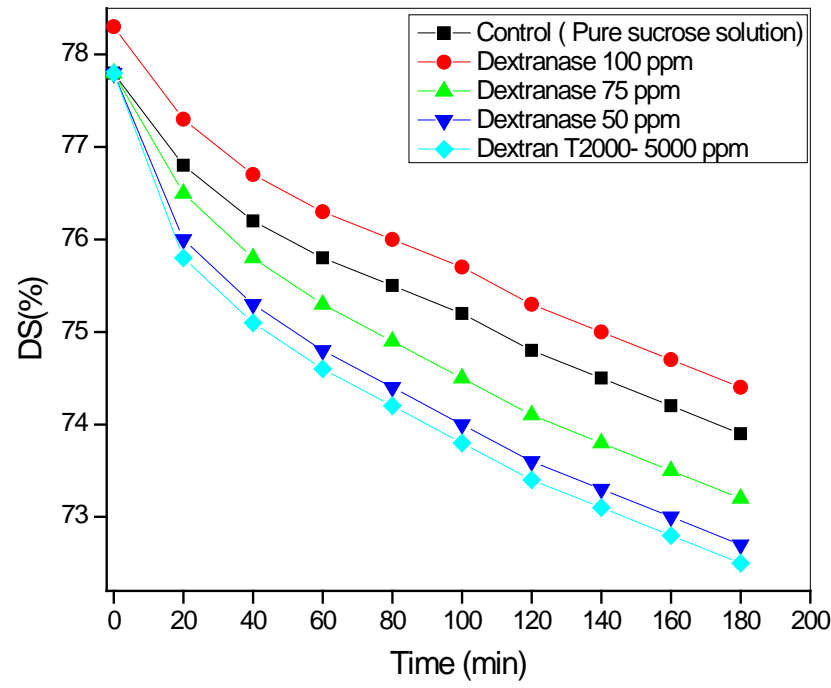

Figure 3. Dry substance content at $60^{\circ} \mathrm{C}$ after hydrolysis of dextran $\mathrm{T}_{2000}$

\subsection{Surface Topography of Sucrose Crystals}

Microscopic examinations of the different faces of sucrose crystals were performed by scanning electron microscopy (SEM). Figure 4 shows the surface topography of a sucrose crystal which was grown in pure sucrose solution at $60^{\circ} \mathrm{C}$. It was observed that the crystal shape has taken a normal form of a sucrose crystal. On the other hand, the microscopic examination of a crystal surface shows the fine sugar crystal on the crystal surface, which developed after the centrifugation process and during the drying operation. The influence of dextran molecules $\mathrm{T}_{2000}$ on sucrose crystal morphology and adsorption surface is shown in Figure 5. It was observed that in presence of this high molecular fraction of dextran the number of conglomerated crystals (multiple crystals where two or more crystals have grown together) increased. A scanning electron micrograph of a sugar crystal shows micro-particles (sucrose crystals) strongly adsorbed on the crystal surface, which cause a rough crystal surface with a number of gaps. Consequently, nonsugar and colorant particles can be adsorbed with dextran on the surface during the technical crystallization process in the sugar factories. Also in this case, the removal of impurities (non-sugars) is difficult during the washing of the crystals. An increase in washing time of the centrifugals is needed to get the required quality of sugar, increasing total centrifuging and purging time. Also, the 
needle-shaped crystal reduces the purging efficiency of the massecuites in the centrifugals resulting in a poor separation of crystal and molasses, hence reducing the refined quality of the sugar [7]. Additionally, dust production was mainly due to the breaking and crushing of conglomerates and needle crystals near to the screens of centrifugals. It has been reported that the increase of sugar crystallization time causes the massecuites to become cooler than normal which increases the already abnormally high viscosity of the fluid [21,22].

Consequently, sugar fine corn in the final product is increased. Such micro-particles may be seen on the surface of a sugar crystal in Figure 5. The conglomerates contain higher ash than unconglomerated sugar. The higher ash is contained in trapped mother liquor, either partially dried in the re-entrant angles, or in the inclusions in those angles.

The effect of crystallization temperature on the surface topography after hydrolysis of dextran by dextranase, at different temperatures was studied (Figure 6). It is obvious that after dextran is hydrolysed by dextranase, more perfect crystal surfaces are built than at $60^{\circ} \mathrm{C}$. As a possible reason, the increase of viscosity caused by dextran and the effects of hydrolysis of high molecular weight dextran on the viscosity of dextran- sucrose solutions can be suggested. Also in crystal topography, the influence of dextran seems to be diminished by the application of higher concentrations of dextran as it could be observed for crystal growth rates. This effect can also be correlated to the increase of colorant and turbidity components of sugar produced at low temperatures (after product). Additionally, the presence of dextran can assist the adsorption of these particles on the crystal surface. Rogé et al., reported that there is a good correlation between white sugar turbidity and calcium ion concentration in stored syrups [2]. As well they reported that macromolecular organic substances (as for example dextran) may act as a carrier for components causing turbidity. Therefore, hydrolysis of dextran before the crystallization process may lead to enhance the crystal rate and decrease the fine sugar crystal on the crystal surface. On the other hand, the number of conglomerated crystals (multiple crystals where two or more crystals have grown together) decreased as can be seen in Figure 6.
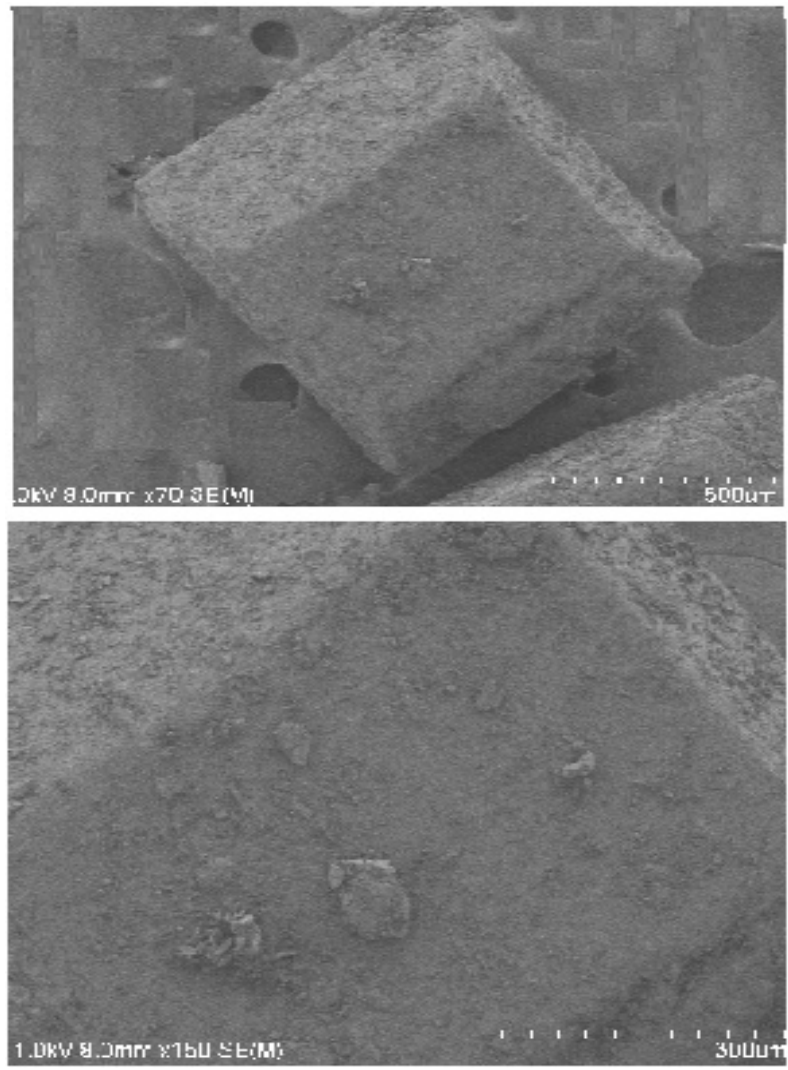

Figure 4. Surface topography of a sucrose crystal grown in the pure sucrose solution
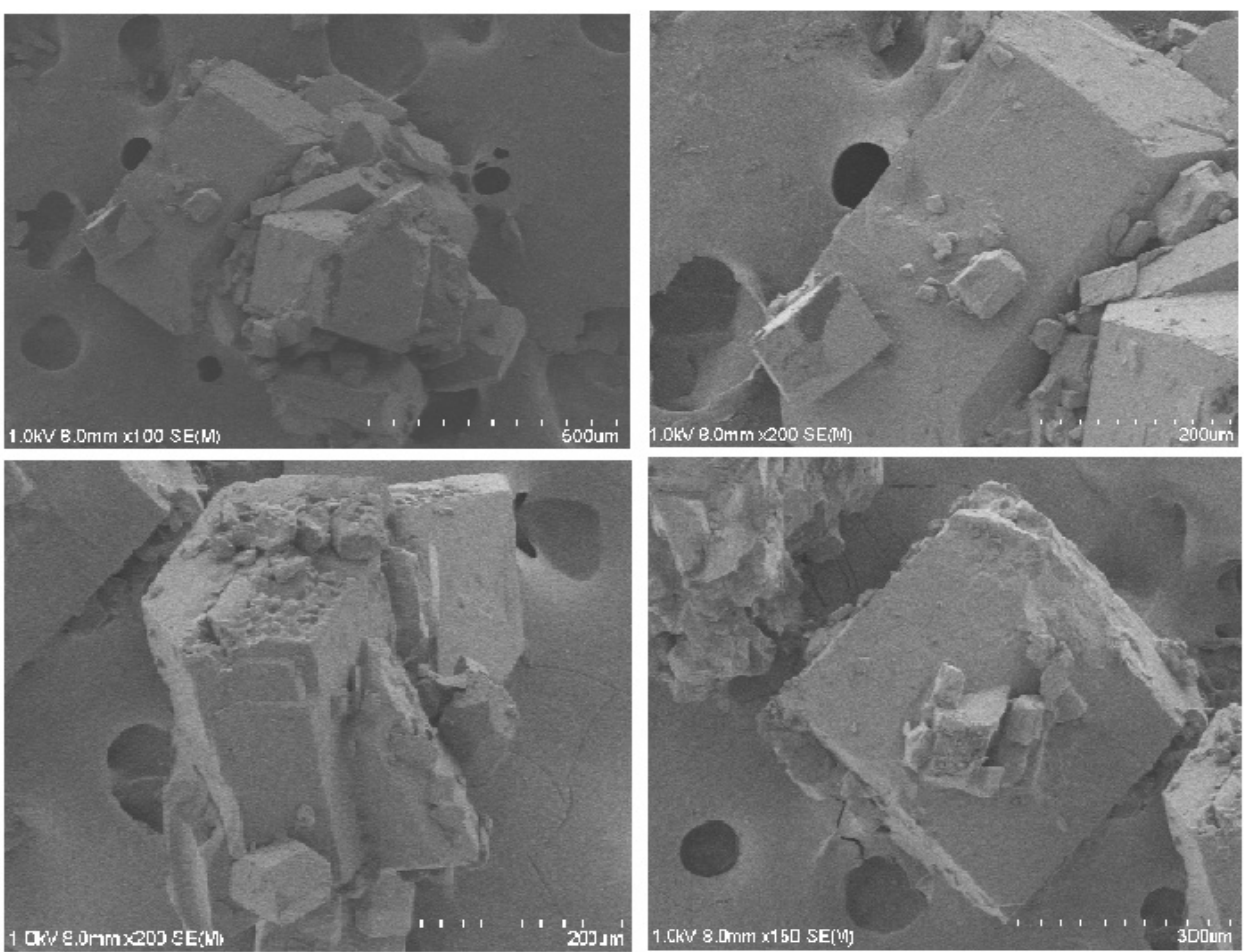

Figure 5. Surface topography of a sucrose crystal grown in the presence of $5000 \mathrm{mg}$ dextran $\mathrm{T}_{2000}$ at $60^{\circ} \mathrm{C}$ 

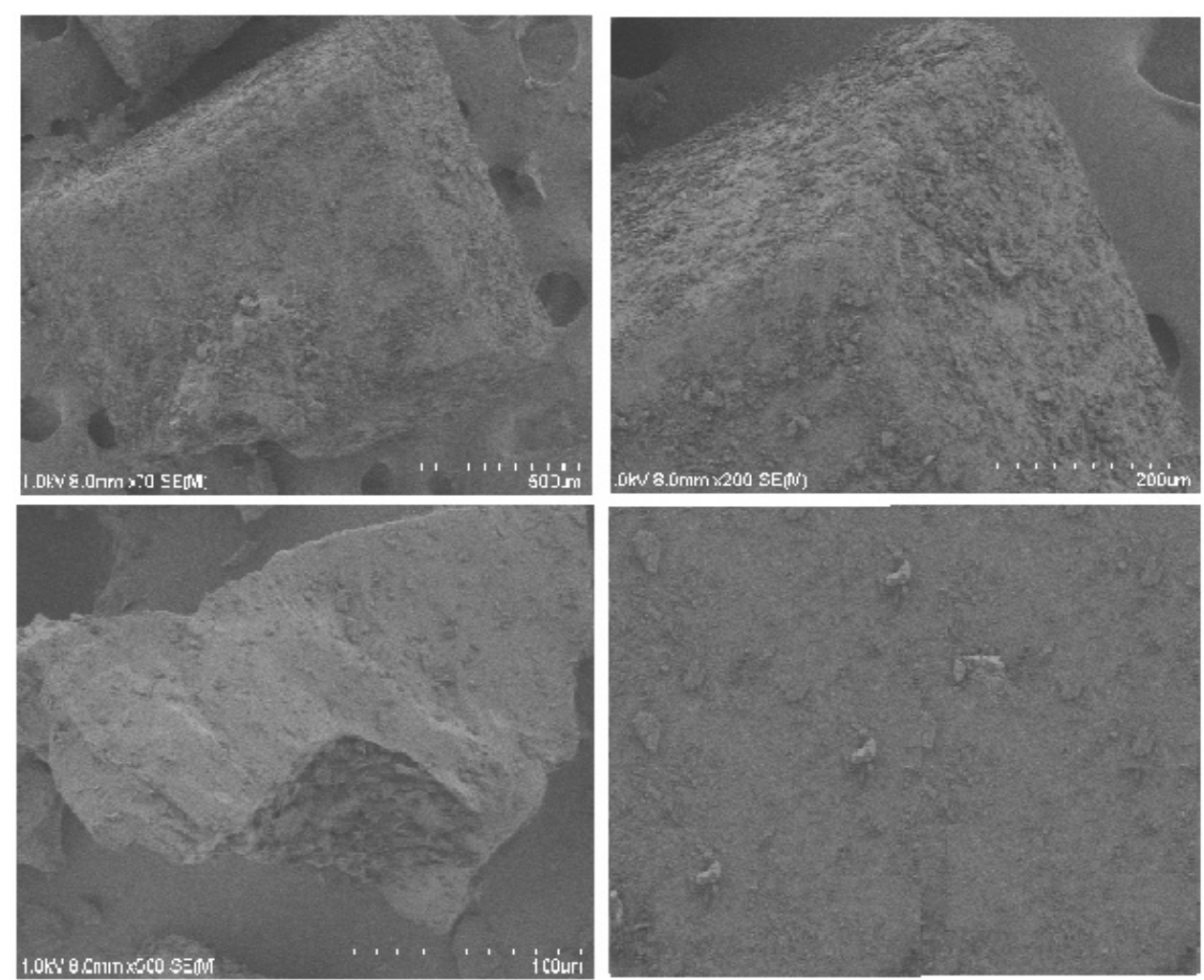

Figure 6. Surface topography of a sucrose crystal grown after hydrolysis of dextran $\mathrm{T}_{2000}$ by dextranase enzyme at $60^{\circ} \mathrm{C}$

In many factories, despite the use of ion exchange to reduce calcium ion, turbidity phenomena in the produced sugar were observed. In addition, a decrease of elongated crystals in the after product crystallization step was observed after hydrolysis of dextran. It has been reported that the elongation of face $\mathrm{c}$ is significant and such a phenomenon increases with syrup turbidity. From the technical sucrose crystallization point of view, the three stage crystallization scheme is regarded as the standard scheme. The process of producing sugar is through several stages, because the high concentration of non-sugar and the increase of solution viscosity hinder the sugar separation from the mother liquor [2].

\section{Conclusions}

In this work, we investigated the influence of the biodegradation of dextran catalyzed by dextranase enzymes during sugar manufacturing on the rate of sucrose crystallization and growth rate of sucrose crystals in pure sucrose solution at different temperatures. To elucidate the influence of biodegradation of dextran on the growth rate of sucrose crystals, dextran of Mw 2,000,000 g/mol ( $\left.\mathrm{T}_{2000}\right)$, were admixed in concentrations between (1000 - 10000 ppm) with $(60 \%$ - 75\% w/w) sucrose solution. The most pronounced effect of dextran on the growth rate of sucrose crystals was found with $\mathrm{T}_{2000}$ at concentrations more than $5000 \mathrm{ppm}$ at $60^{\circ} \mathrm{C}$. From the results it could be shown that an increase of crystallization rate of up to $50 \%$ after biodegradation of dextran using dextranase enzyme at 100 ppm, compared to crystallization rate with pure sucrose solution in the presence of dextran $T_{2000}$. Such a positive influence of biodegradation of dextran using dextranase enzyme decreases crystallization time in the sugar house and thus decreases the production costs of sugar manufacturing.

\section{References}

[1] Meade, G. P. and Chen, J. C. P. (1977). Cane Sugar Handbook, John Wiley and Sons, New York.

[2] Rogé, B., Bensouissi, A. and Mathlouthi, M. (2007). Effect of calcium on white sugar turbidity. Zuckerindustrie, 132(3), 170-174.

[3] Khalikova E., P. Susi, T. Korpela. (2005). Microbial dextranhydrolyzing enzymes: Fundamentals and applications. Microbiol Mol Biol Rev 69(2): 306-324.

[4] Cuddihy J.A., and D.F. Day. 1999. The process and financial impact of dextran on sugar factory. [cited 13 May 2018], Available from: http://www.midlandresearchlabsinc.com.

[5] Aquino, W. F., Franco, W. D. (2009). Molecular mass distribution of dextran in Brazilian sugar and insoluble deposits of cachaça. Food Chemistry 114:1391-1395.

[6] Kim D. and D.F Day. 2004. Determination of dextran in raw sugar process streams, Food Science and Biotechnology. 13, 248-252.

[7] Abdel-Rahman E.A., Q. Smejkal, R. Schick, S. El-Syiad, T. Kurz. (2008). Influence of dextran concentrations and molecular fractions on the rate of sucrose crystallization in pure sucrose solution. J Food Eng 84, 501-508.

[8] Eggleston G, Côté G, Santee C. (2011). New insights on the hardto-boil massecuite phenomenon in raw sugar Manufacture. Food Chemistry; 126, 21-30.

[9] Bashari, M., Eibaid, A., Wang, J., Tian, Y., Xu, X. and Jin, Z., (2013). Influence of low ultrasound intensity on the degradation of dextran catalyzed by dextranase, Ultrasonics Sonochemistry. 20, 155-161.

[10] Bashari, M., Abdelhai, M. H., Abbas, S., Eibaid, A., Xu, X., \& Jin, Z.. (2014). Effect of ultrasound and high hydrostatic pressure (US/HHP) on the degradation of dextran catalyzed by dextranase. Ultrasonics sonochemistry. 21, 76-83.

[11] Bashari, M., Jin, Z., Wang, J., \& Zhan, X. (2016). A novel technique to improve the biodegradation efficiency of dextranase enzyme using the synergistic effects of ultrasound combined with microwave shock. Innovative food science \& emerging technologies 35: 125-132. 
[12] Bashari, M., Nikoo, M., Jin Z., Bai, Y., Xu, X., Yang, N. (2012). Thermal and rheological properties of the supersaturated sucrose solution in the presence of different molecular weight fractions and concentrations of dextran, Eur Food Res and Techno 234(4), 639-648.

[13] Braga da Cruz, I., MacInnes, W. M., Oliveira, J. C., \& Malcata, F. X. (2002). Supplemented state diagram for sucrose from dynamic mechanical thermal analysis. In H. Levine (Ed.), amorphous food and pharmaceutical systems (pp. 59-70). Cambridge: The Royal Society of Chemistry.

[14] Lowary, T. L., \& Richards, G. N. (1988). Effects of impurities on hydrolysis of sucrose in concentrated aqueous solution. International Sugar Journal, 90(1077), 164-167.

[15] Quintas, M., Lobo, L., Ribeiro, L., \& Silva, C. L. M. (2001) Kinetics of high temperature degradation of concentrated sucrose solutions. Poster presented at EUROCAFT, Berlin, Germany.

[16] Clarke M.A., J. Bergeron, and F. Cole. 1987. A rapid dextran screening test. Sugar Azucar 82(3): 23-24.

[17] Austmeyer, K. E. (1981). Untersuchungen zum Wärme-und
Stoffübergang im Anfangsstadium der Verdampfungskristallisation der Saccharose, Techn. Univ. Carlo-Wilhelmina zu Braunschweig. Germany

[18] Bashari M., A. Eibaid, J, Wang, Y, Tian, X. Xu, Z. Jin. 2013 Influence of low ultrasound intensity on the degradation of dextran catalyzed bydextranase, Ultrason Sonochem 20, 155-161.

[19] Bashari, M., Tounkara, F., Abdelhai, M. H., Lagnika, C., Xu, X. \& Jin, Z. (2013). Impact of dextranase on sugar manufacturing and its kinetic on the molecular weights of remaining dextran. Sugar Tech, 15(1), 84-93.

[20] Ekelhof, B. (1997). Kristallisationskinetik der Saccharose in reinen und unreinen Lö. sungen. Dissertation, Techn. Univ. Braunschweig, Germany.

[21] Cuddihy, J. A., Miguel, E. P. and James, S. R. (2000). The presence of total polysaccharides in sugar production and methods for reducing their negative effects. Midland Research Laboratories, Inc.

[22] Jimenez, E. R. (2005). The dextranase along sugar-making industry. Biotecnologia Aplicada, 22(1), 20-27.

(C) The Author(s) 2019. This article is an open access article distributed under the terms and conditions of the Creative Commons Attribution (CC BY) license (http://creativecommons.org/licenses/by/4.0/). 